\title{
Forecasting Commodity Price Index of Food and Beverages in Kenya Using Seasonal Autoregressive Integrated Moving Average (SARIMA) Models
}

\author{
Teddy Mutugi Wanjuki, Adolphus Wagala, and Dennis K. Muriithi
}

\begin{abstract}
Price stability is the primary monetary policy objective in any economy since it protects the interests of both consumers and producers. As a result, forecasting is a common practice and a vital aspect of monetary policymaking. Future predictions guide monetary and fiscal policy tools that that be used to stabilize commodity prices. As a result, developing an accurate and precise forecasting model is critical. The current study fitted and forecasted the food and beverages price index (FBPI) in Kenya using seasonal autoregressive integrated moving average (SARIMA) models. Unlike other ARIMA models like the autoregressive (AR), Moving Average (MA), and non-seasonal ARMA models, the SARIMA model accounts for the seasonal component in a given time series data better forecasts. The study relied on secondary data obtained from the KNBS website on monthly food and beverage price index in Kenya from January 1991 to February 2020. R-statistical software was used to analyze the data. The parameter estimation was done using the Maximum Likelihood Estimation method. Competing SARIMA models were compared using the Mean Absolute Error (MAE), Mean Absolute Scaled Error (MASE), and Mean Absolute Percentage Error (MAPE). A first-order differenced SARIMA $(1,1,1)(0,1,1) 12$ minimized these model evaluation criteria $($ AIC $=1818.15$, BIC $=1833.40$ ). The forecasting ability evaluation statistics MAE $=2.00 \%$, MAPE $=1.62 \%$ and MASE $=0.87 \%$. The 24-step ahead forecasts showed that the FPBI is unstable with an overall increasing trend. Therefore, the monetary policy committee ought to control inflation through monetary or fiscal policy, strengthening food security and trade liberalization.
\end{abstract}

Keywords - Consumer price index, forecasting, modelling. monetary policy, SARIMA models, price stability.

\section{INTRODUCTION}

The primary goal of monetary policy making and implementation is price stability. With the fluctuation in oil prices due to the supply forces brought in by the OPEC and non-OPEC nations, other commodity prices are likely to fluctuate [1]. Reference [2] found that food and oil price shocks contribute to Kenya's persistent inflationary pressures. The World Bank's commodity markets outlook in 2019 showed an increasing trend of commodity prices like oil and agricultural produce and are likely to peak on average by 2020. Food and beverage price fluctuation in Kenya has been attributed to seasonal fluctuations in food production. Frequent and unpredictable changes in prices is a cause of concern since it affects investment and consumer behavior.

While high prices may benefit the producers, owing to supernormal profits, it can indicate high volatility of prices and hence uncertainty on future prices. Such fluctuations in the market discourage both local and foreign investments in the commodity market. To the consumers, high market prices adversely affect their standards of living. Consequently, increased price volatility may exacerbate the country's balance of payments problems and depress economic growth. On the contrary, low export prices may cause the balance of payments to deteriorate. Moreover, deflation may impact the economy because it lessens firms' profitability, which is a disincentive investment. As a result, levels of prices must be stabilized in a way that encourages growth in the economy. Overall, uncertainty about future prices may dampen investment and repress a country's economy's growth [3], [4].

As a key feature of commodity markets, price volatility necessitates forecasting future trends in order to make an informed investment strategies and government policies aimed at price regulation [5]. Monitoring

Published on December 21, 2021.

T. M. Wanjuki, Chuka University, Kenya.

(corresponding e-mail: wanjukiteddymutugi@gmail.com)

A. Wagala, Bomet University College, Kenya.

(e-mail: wagala@buc.ac.ke)

D. K. Muriithi, Chuka University, Kenya.

(e-mail: kamuriithi2011@gmail.com) 
commodity price indices aids in the development of evidence-based consumption and investment reforms. Besides, consumers and investors who use some commodities as inputs in their production processes need to understand the nature and trend of commodity prices to make informed decisions concerning the future. In Kenya, the monetary policy committee controls inflation and implements price stability policies. The Central Bank of Kenya (CBK) keeps market prices stable to avoid inflation [6]. To mitigate the unexpected surge and adverse impact of inflation, the central banks employ the inflation forecast targeting (IFT) or inhouse Forecasting and Policy Analysis Systems (FPAS), which are forward-looking regimes where inflation forecast is the intermediate target [7]. The CBK has an inflation aim range of 2.5 percent and 7.5 percent [8]. The FPAS framework utilizes the simple quarterly projection model that incorporates uncertainty and moral suasion. Deviation from the target helps formulate restorative policy decisions to bring the inflation forecasts on track [9].

Time series modeling approaches have grown in popularity in modeling time series due to increased demand for forecasts in economic time series data. Since seasonal or periodic fluctuations in commodity prices are a common feature in the commodity market, Economists and Statistics face the challenge of getting the best predictive models to be used in forecasting. Additionally, the non-linearity in commodity prices, such as food and beverages, necessitates non-linear models in its prediction. The Seasonal Autoregressive Integrated Moving Average

(SARIMA) model, unlike other ARIMA models like the autoregressive models (AR), Moving Average (MA), and ARMA models, accounts for seasonal variation in a given time series data. The SARIMA has been useful in many fields finding its applicability in modeling and forecasting series exhibiting seasonality such revenues forecasting [10], unemployment forecasting [11], inflation in Ghana [12], tomato prices in Turkey [13], rainfall in Kenya [14], annual sugarcane yields data in India [15], exchange rate forecasting [16], and inflation forecasting [17], the unemployment rate in Greece [18], and tomato wholesale prices [19]. The current study fitted the SARIMA model monthly food and beverage prices in Kenya from 1991 to 2020 .

\section{LITERATURE REVIEW}

The SARIMA models have been widely used to model inflation data in several economies. The study of [20] empirically modeled and forecasted monthly inflation data in Ghana applying monthly based data from January 1990 to January 2012. The ARIMA $(3,1,3)(2,1,1) 12$ model order was the best fit in modeling the inflation data. The model minimized the prediction errors were based on MAPE of 1.902, RMSE of 0.080 , and MAE of 0.054. Reference [21] discovered that the SARIMA $(0,1,1)(0,1,1) 4$ model was the best fit for tomato prices in Ghana's Ashanti region $(\mathrm{BIC}=5.969)$. They observed significant price fluctuations in tomato prices throughout the year. Prices can often increase tenfold during peak harvest seasons, implying that if farmers plan their tomato areas, planting dates and sales dates by evaluating predicted rates from the fitted ARIMA models, they will obtain inflated prices, profitability could rise at least by three to fourfold with 90 percent certainty of the prediction accurateness.

Reference [22] conducted a study in which they used SARIMA Models to forecast rates of inflation in Nigeria form the year 2003 (November) to 2013 October with 120 observations. They discovered that the SARIMA $(1,1,1)(0,0,1) 12$ model was the most accurate at forecasting Nigeria's inflation rate $($ AIC $=$ 5.4813 , BIC = 5.5742). Reference [17] used quarterly data from 1981 to 2013 to forecast the Kenyan inflation rate using SARIMA. By minimizing the Akaike Information Criterion value, the researchers discovered that SARIMA $(0,1,0)(0,0,1) 4$ was the most considered model for predicting inflation rates in Kenya. The predictive ability tests of the model were based on minimization of predictions errors using several metrics, including the MAPE $=3.945$, RMSE $=0.2871$, and MAE $=0.2369$, which depicted that the used model was the most suitable for predicting the rates of inflation in Kenya. Using Kenya's inflation rate from 1981 and 2013, Gikungu et al [23], identified SARIMA $(0,1,0)(0,0,1) 4$ as the appropriate model to fit the data. The predictability tests, $\mathrm{MAPE}=3.945, \mathrm{RMSE}=0.2871$, and $\mathrm{MAE}=0.2369$, demonstrated that the resultant model was suitable for predicting inflation rates in Kenya.

The study of [24] contracted the suitability of the SARIMA and holt-winters (HW) approach in fitting short-term inflation in Ghana applying data on monthly inflation for January 1971 to 2012 October. The study findings of the SARIMA forecast from ARIMA $(2,1,1)(0,0,1) 12$ presented the prediction metrics as follows mean absolute error (0.1787), root mean square error (0.2104), mean absolute percentage error (1.9123) and mean absolute scaled error (0.0073) and the Seasonal Multiplicative HW forecast was MAE (2.2305), RMSE (2.4274), MAPE (24.000), MASE (0.0911). Thus, the study proposed the SARIMA model as the best fit for the short-term prediction method for inflation in Ghana. The performance of Holt-Winters Triple Exponential Smoothing and SARIMA models were also compared by [25] using monthly Kenya's inflation data between November 2011 and October 2016. The study findings indicated that the SARIMA model $(\mathrm{MASE}=0.059, \mathrm{MAE}=0.004, \mathrm{MAPE}=0.073)$ is the best candidate model for the inflation data 
than HW Triple Exponential Smoothing model (MASE = 0.643, MAE 0.595, MAPE =0.400). In Ghana, [26] examined the monthly inflation data from January 2010 to September 2017 with 104 data points. ARIMA $(1,1,1)$ was the most suitable model that minimized the prediction errors $(\mathrm{RMSE}=1.163)$, outperforming competing orders.

Overall, the reviewed studies have applied Box and Jenkins techniques of time series modeling using SARIMA. Most found that it is the most suitable model for modeling time series data characterized by seasonality and non-stationarity. However, the context of the reviewed studies presents gaps to be the field in the current study. First, the study was conducted in a different economy, unlike the current study, which focuses on Kenya's economy. Besides, most of the reviewed considered the general price level, unlike the current study, which focuses explicitly on Kenya's food and beverages price index.

\section{DATA AND METHODS}

\section{A. Data}

The study does not involve any probabilistic sampling procedures since it is a time series analysis. The data used was monthly food and beverage prices index data in Kenya for the period January 1991 to February 2020 extracted from Kenya's KNBS (knbs.or.ke). The selected time frame has 350 data points and is thus sufficient for time series modeling. The chosen period is also considered appropriate economically since the period includes the economic shocks such as the post-election violence in Kenya of 1997, 2002, and 2007 and the 2007/08 global recession. Such shocks are responsible for the seasonal shocks to the consumer price index that SARIMA models capture.

\section{B. Seasonal Autoregressive Integrated Moving Average Models}

SARIMA models are an adjustment of Autoregressive Integrated Moving Average (ARIMA) models that accurately model seasonal time series data. Seasonality is defined in time series as a systematic pattern of repeated fluctuations over a given time, given $\mathrm{h}$ is the periodicity of the series [27]. For example, data observed quarterly has $\mathrm{h}$ being 4 . This behaviour, which is frequent in most time series data, renders the Autoregressive Integrated Moving Average model ineffective when applied to the series. Seasonal AR and MA terms in an ARIMA forecast $X_{t}$ based on previous observations and residual errors with lags multiples of S. (the seasonality duration of the series). The ARIMA models (non-seasonal) are widely referred to as ARIMA ( $p, d, q)$, given $p$ is the order for AR model denoting the set of time lag(s), $d$ gives the differencing order, and q the MA order of the model [28]. The seasonal ARIMA model, on the other hand, combines non-seasonal and seasonal components represented as ARIMA (p,d,q) (P, D, Q)h [29].

Let $B^{S}$ represent the operator then we have,

$$
B^{S} X_{t}=\left(X_{t}-X_{t-s}\right)
$$

The seasonal differencing is denoted as,

$$
\left(1-B^{S}\right) X_{t}=\left(X_{t}-X_{t-s}\right)
$$

which can be obtained using Equation (3considering data observed monthly, we have $s=12$

$$
\begin{aligned}
& \left(1-B^{12}\right) X_{t}=\left(X_{t}-X_{t-12}\right) \\
& \quad\left(1-B^{12}\right) X_{t}=\left(X_{t}-X_{t-12}\right)
\end{aligned}
$$

SARIMA model, which is characterized by both seasonal order (P,D,Q) and non-seasonal order (p,d,q), is simplified as SARIMA $(\mathrm{p}, \mathrm{d}, \mathrm{q})(\mathrm{P}, \mathrm{D}, \mathrm{Q})$ s model and can be composed as

$$
\varphi(B) \Phi\left(1-B^{S}\right)^{D} X_{t}=\theta(B) \Theta\left(B^{S}\right) Z_{t}
$$

In that $\Phi$ and $\Theta$ imply polynomials in $B^{S}$ of order $\mathrm{P}, \mathrm{Q}$ respectively. The SARIMA $(0,1,1)(0,1,1){ }_{12}$ is one of the commonly used model that is especially for seasonal data. Reference [30] confirm that [31] SARIMA model is presented by (5).

$$
\varphi_{P}\left(B^{S}\right) \Phi(B) \nabla_{S}^{D} \nabla^{d} x_{t}=\alpha+\Theta_{Q}\left(B^{S}\right) \theta(B) Z_{t}
$$

where $Z_{t}$ denotes the gaussian white noise sequence. The ARIMA (p d q) (P, D, Q) 12 is the general model. The autoregressive (AR) and moving average (MA) parts are depicted by polynomials $(\Phi)$ and $\theta$ of orders $\mathrm{p}$ and $\mathrm{q}$ respectively while seasonal autoregressive (SAR) and seasonal moving average (SMA) parts are 
denoted by $\varphi_{P}\left(B^{S}\right)$ and $\Theta_{Q}\left(B^{S}\right)$ of orders $\mathrm{P}$ and $\mathrm{Q}$, respectively and the differencing components for both ordinary and seasonal are denoted by;

$$
\begin{aligned}
\nabla^{d} & =(1-B)^{D} \\
\nabla_{S}^{D} & =\left(1-B^{S}\right)^{D}
\end{aligned}
$$

Given the data of interest is monthly data, $\mathrm{s}=12$, generally, the multiplicative SARIMA model becomes;

$$
(1-B)\left(1-B^{12}\right) X_{t}=(1+\theta B) \Theta\left(B^{12}\right) Z_{t}
$$

Under this approach, CPI data with monthly observations per year was employed. The first order SAR(1) model used $X_{t-12}$ in predicting $X_{t}$, whereas the SMA (1) used $Z_{t-12}$ as it predictor.

\section{Data Analysis}

R-Statistical Software [32] was used to analyze the data. The preliminary steps involved determining the data's stationarity. SARIMA models are suitable for stationary time series with steady mean and variance over time. Data exploration was done by examining the visual plot to check for any underlying patterns of behavior. The scatter points have a constant mean and variance for stationary time-series data and oscillate around the mean. However, for a non-stationary time series, the time plot indicates an upward or downward trend which implies non-constant mean and variance. The data's stationarity was assessed applying the popular Augmented Dickey-Fuller (ADF) method developed by [33]. The ADF technique tries to preserve the reliability of the test on the bases of white noise in the regression model by guaranteeing that the noise is indeed white. Assume the ADF statistic is larger than the critical value at a 5\% level of significance. In that case, the null hypothesis is that a unit root is recognized and non-stationary is confirmed in the series. For a non-stationary series, differences at various degrees were performed to obtain a stationary series. According to [34], the modeling approach comprises of four stages: model identification, estimation of the model, diagnostic checking, and model forecasting, discussed as follows;

\section{1) Model Identification or Selection}

The model selection criteria entail finding the most suitable model to fit the data by finding a model that neither underestimates nor overestimates the data. Reference [35] state that the principle of parsimony is applied where a model with few parameters is preferred. The first step of modeling is detecting the appropriate SARIMA ( $\mathrm{p}, \mathrm{d}, \mathrm{q})(\mathrm{P}, \mathrm{D}, \mathrm{Q})$ order of the model. The order specification and selection of orders $\mathrm{p}$ and $\mathrm{q}$ entail plotting the sample correlogram at various lag lengths, and the order $\mathrm{d}$ is approximated using the I (1) or I (0) process [36]. Considering the non-seasonal behavior, the sample autocorrelation function (ACF) and partial autocorrelation function (PACF) typically have spikes at lag k and cut off after lag k. ACF and PACF have spikes at lag ks and cut off after lag ks at the seasonal level. The number of significant spikes indicates the model's order [37] Tables I and II show how the ACF and PACF behave for both seasonal series and non-seasonal series [30]. The differencing $\mathrm{d}$ and $\mathrm{D}$ order is determined by differencing order performed in making the series stationary.

TABLE I: ACF AND PACF BEHAVIOUR FOR NON-SEASONAL ARIMA (P, Q)

\begin{tabular}{cccc}
\hline \hline & AR $(\mathrm{p})$ & MA $(\mathrm{q})$ & ARMA(p,q) \\
\hline \hline ACF & Tail off at lag k & Cuts off at lag q & Tails off \\
PACF & Cuts off at lag p & Tails off at lag $\mathrm{k}$ & Tails off \\
\hline \hline & & & \\
& & & \\
\hline \hline & TABLE II: ACF AND PACF BEHAVIOUR FOR THE SEASONAL ARIMA (P, Q) & ARMA $(P, Q)_{s}$ \\
\hline ACF & AR(P) & MA(Q) & Tails off \\
PACF & Tail off at lag Ks & Cuts off at lag Qs & Tails off \\
\hline \hline
\end{tabular}

The current study used Akaike Information Criterion [38] and the Bayesian Information Criterion to select a parsimonious model. A model which minimizes these information criteria was chosen. Whereas AIC attempts to measure the model to the certainty of the state, BIC seeks the best [39]. The AIC is computed using equation (8) [38].

$$
A I C=\left(\frac{E S S}{N}\right) e^{\frac{2 f}{n}}
$$

where; $n$ denotes the number of observed values, $f$ represents parameters under consideration, and, ESS is the Error sum of squares.

The BIC is obtained using equation (10) [40] 


$$
B I C=-2 \log (L)+k \log (n)
$$

The $\mathrm{k}$ denotes parameters in the model, $(\mathrm{p}+\mathrm{q}+\mathrm{P}+\mathrm{Q}+1)$, $\mathrm{L}$ denotes the maximum likelihood function value for the proposed model and $\mathrm{n}$ gives the size of sample.

\section{2) Model Estimation}

The lagged observations of the error terms are unobservable. Thus, the maximum likelihood estimation (MLE) method is preferred in estimating parameters of the model instead of the Ordinary Least Squares (OLS) regression method since it is not possible to derive explicit expressions to estimated unknown coefficients as opposed to AR models where we utilize lagged values of a given series. For a T observations sample, the log-likelihood (LL) function of $\left\{\mathrm{y}_{-} \mathrm{t}(\theta)\right\}$ following the premise of independent and distributed standardized 9, is denoted by:

$$
\operatorname{Ln} L\left[y_{t}(\theta)\right]=\sum_{t=1}^{T}\left[\ln \left[D\left(Z_{t}(\theta), v\right)\right]-\frac{1}{2}\left[\ln \left(\sigma^{2}{ }_{t}(\theta)\right]\right]\right.
$$

Where; $\theta$ is a vector of the conditional variance, conditional mean, and density function 9 parameters that must be estimated, and is a sequence of random variables with a mean of zero and variance one.

The estimated parameters of $\theta$ can be estimated using a multivariate normal distribution with its mean given and covariance matrix usually denoted by:

$$
V_{n}=\frac{1}{n}\left(\operatorname{Var}\left[V_{\theta} \ln \left(f_{x}\left(X ; \theta_{0}\right)\right)\right]\right)^{-1}
$$

where:

$\ln \left(f_{x}\left(X ; \theta_{0}\right)-\log\right.$-likelihood estimated at the parameter $\theta_{0}$; $V_{\theta} \ln \left(f_{x}\left(X ; \theta_{0}\right)-\right.$ vector of the first derivatives.

\section{3) Model Diagnostics}

In an ideal situation, a model would extract all methodical information from the data. Model diagnostic checks are typically based on the model's residuals $\left(e_{-} t\right)$ and regulate the suitability of the chosen model. Among the premise of the ARIMA class of models is that the model's residuals ought to be white noise. If e_t are independently and identically distributed with zero mean and a variance $\llbracket \sigma \rrbracket \_t$, the series is a gaussian white noise [41]. For whiteness, the ACF is all zero. In practice, the autocorrelation function of the noise is close to zero if the model's residuals are indeed white noise. To test for homoscedasticity and normality, the residual's visual plot and Q-Q plots were used. The Ljung Box Q statistic was employed to establish if the residuals errors are independent. The Box-Pierce $\mathrm{Q}$ statistics is computed using equation (14) [41].

$$
Q_{m}=n(n+2) \sum_{k=1}^{m} \frac{e_{k}^{2}}{n-2}
$$

where $e_{k}$ denotes the autocorrelation of residuals at lag $k$, $n$ represent the number of residuals,

$m$ denotes the time lags.

The model is considered adequate if the probability value associated with the Ljung-Box Q statistic is greater than the critical level of significance. The correlogram of residuals be used to test the autocorrelation. For non-correlated series, the ACF and PACF should be close to zero for all lags, and the Q-Statistics would be trivial with larger p-values [18].

\section{4) Model Accuracy Evaluation}

To determine model performance in terms of in-sample forecast, the precision of competing models can be checked. Some observations are omitted during model construction in out-of-sample forecasting. The current study employs three model evaluation metrics: MAE, RMSE, and MAPE, to test accuracy of the model. The model presenting the lowest number of these metrics is thought to be a better fit for use in forecasting. If the forecast is perfect all the aforementioned metrics equal to zero. The lower the value, the more accurate forecasts are and vice versa. The model forecasting ability depends entirely on minimization of the accuracy metrics. The RMSE calculates the difference or residuals between the model's forecasts and observed values (14). 


$$
R M S E=\sqrt{\frac{E S S}{n}}
$$

The "mean prediction error" is measured by RMSE. The RMSE will be zero for a "Perfect" fit. Where; ESS is the error sum of squares:

$$
E S S=\sum_{t=1}^{n}\left(\hat{y}_{t}-y_{t}\right)^{2}
$$

which implies that

$$
R M S E=\sqrt{\frac{\sum_{t=1}^{n}\left(\hat{y}_{t}-y_{t}\right)^{2}}{n}}=\sqrt{\frac{\sum_{t=1}^{n} e_{t}^{2}}{n}}
$$

The MAE is obtained using (17).

$$
M A E=\frac{\sum_{t=1}^{n}\left|Y_{t}-\hat{Y}_{t}\right|}{n}
$$

where, $Y_{t}$ :actual observed value at time t;

$\hat{\mathrm{Y}}_{t}$ : forecasted observations;

$n$ : observations.

\section{5) Forecasting Using SARIMA}

Forecasting is the final stage in the Box and Jenkins' model building approach. Forecasting inflation is a common practice and a vital input when making monetary policy decisions [43]. It is very important in the planning and decision making. It aids in anticipating future uncertainty on the bases of previous and current observations' behavior. After passing the diagnostic test, the model is ready for forecasting. For example, given the SARIMA $(0,1,1)(1,0,1) 12$, the next step ahead forecasts are as follows:

$$
\begin{aligned}
& Z_{t}-Z_{t-1}=\Phi\left(Z_{t-12}-Z_{-13}\right)+\varepsilon_{t}-\theta \varepsilon_{t-1}-\Phi \varepsilon_{t-12}+\theta \varepsilon_{t-13} \\
& Z_{t}=Z_{t-1}+\Phi\left(Z_{t-12}-Z_{-13}\right)+\varepsilon_{t}-\theta \varepsilon_{t-1}-\Phi \varepsilon_{t-12}+\theta \varepsilon_{t-13}
\end{aligned}
$$

The 1-step ahead forecast of the origin $t$ is as follows:

$$
\hat{Z}_{t+1}=Z_{t}+\Phi\left(Z_{t-11}-Z_{-12}\right)-\theta \varepsilon_{t}-\Phi \varepsilon_{t-11}+\theta \varepsilon_{t-12}
$$

The noise term $\varepsilon_{13}, \varepsilon_{12}, \varepsilon_{11}, \ldots, \varepsilon_{1}$ (as residuals) enters into the predictions for leading times $l=$ $1,2, \ldots, 13$ nonetheless, for $l>13$, the model's Autoregressive component takes over:

$$
\hat{Z}_{t+1}=Z_{t-l+1}+\Phi Z_{t+l-12}-\Phi Z_{t+l-13} \text { for } l>13
$$

\section{RESUltS AND Discussion}

\section{A. Descriptive Statistics}

Table III shows that the least FBPI recorded was 75.83 in 2001M12, and the highest was 265.79 in 2020M2. The series has a higher variability since the standard deviation statistic is large (51.07). This suggests non-stationarity of the data. The intuition relies on the fact that for stationary time series the mean and standard deviation, do not change over time. The skewness statistic of 1.20 , which is positive, implies that the FBPI series has a slightly long-right tail. However, the value is within the recommended range of +-3 ; hence the data is assumed to be approximately normally distributed since the skewness statistic is almost zero. In such a case, the series can be modeled without carrying out a log transformation on the data.

\begin{tabular}{cccccccc}
\multicolumn{7}{c}{ TABLE III: DESCRIPTIVE STATISTICS } \\
\hline Statistic & Min & Mean & Variance & SD & Max & Skewness & Kurtosis \\
\hline FBPI & 75.83 & 130.32 & 2607.95 & 51.07 & 265.79 & 1.20 & 0.26 \\
\hline
\end{tabular}




\section{B. Visualization of the Series}

The visual plot of the food and beverage price index data (see Fig. 1) Indicates the original data set has an upward trend with two notable peaks. The first one occurred in the 1990s, where the 1996 and 1997 had an annual average index of 113.7 and 111.3, respectively. The rise can be linked with the following structural changes in the economy before the peak. In 1993, the dual system where both official rates of exchange and the "market" rate was abolished. The liberalization allowed a flexible rate exchange determined at the market rate. The immense private capital influxes led to the accumulation of foreign exchange balances in the commercial banks. As a result, the excess money supply in circulation amidst the inadequate supply of essential commodities pushed prices up [44]. Treasury bill discount rate to mitigate excess liquidity, which in turn pushed the inflation rate up. The prices started to subside following the depreciation of Kenya's currency in 1998.

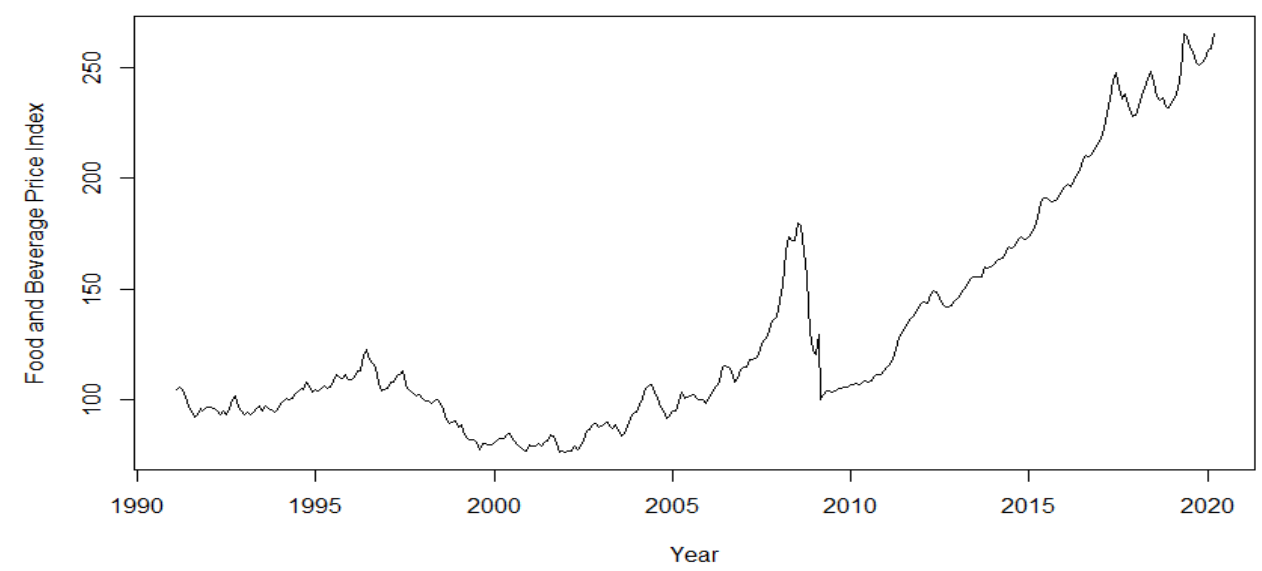

Fig. 1. Time series plot of food and beverage price index.

Note. CPI index before February 2020 is calculated from CPI with base February $1982=100$ and after that calculated with base February 2009=100.

The second period with a sharp rise in food prices as well as oil prices between 2007/8 and 2011 (focusing on the slope since the given visual plot has two base years: 1982 and 2009) can be attributed to the striking external shocks. The shock came shortly after a relatively stable food price during 2007 due to the bumper harvest [45]. The first food crisis of 2008/9 (majorly surging food cereals prices such as rice and wheat) can be associated with the high oil and energy prices, low-interest rates, devaluation of the dollar, poor performance in the agricultural sector due to adverse weather conditions (droughts), and restrictions of rice export by major rice-producing countries as a form of protectionism of the domestic prices following the shortage in supply which in turn lead panic buying of imported grains [9]. The inflated oil prices pushed costs of cereal production up since the agricultural sector is primarily energy-intensive. The shortage in supply was compounded by the rising demand for cereals from wealthy OPEC who benefited from surging oil prices. All these factors skyrocketed food prices across the globe. Overall, there has been an upward in food prices in Kenya, which rose steadily from 2009 to 2020. The trend is not favorable, especially to consumers, since it dilutes their real income.

\section{Decompositon of the Series}

A seasonal time series is made up of three components: a trend component, a seasonal component and an irregular component. Decomposing a time series entails breaking it down into these three main components. Seasonal decomposition was used to evaluate if the series exhibits the three main components. The visual plot in Fig. 2 showed the initial series (observed) with its linked three components (seasonal, random error and trend). Meanwhile, the series features a seasonal component, thus necessitates the use of a model that captures seasonality, in this case, the SARIMA model. 


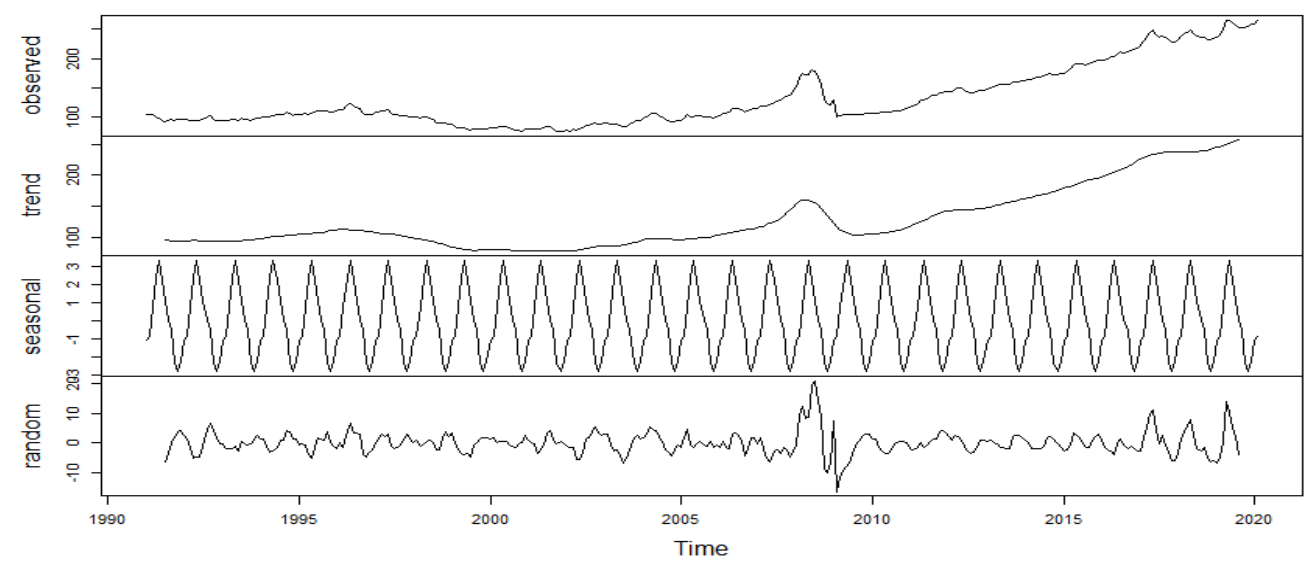

Fig. 2. Decomposition of the Price Index Series.

\section{Testing for Non-stationarity}

Fig. 1 depicts the monthly series of FBPI data evolution. The series does not appear stationary (has a changing mean and variance over time) with notable seasonal fluctuation attributed to external shocks (oil prices and exchange rate movements; weather conditions) and internal shocks (political instability and monetary policy). The study used ADF unit roots tests to perform a statistical test for series stationarity [33]. Table IV shows the Augmented Dickey-Fuller (ADF) test results on the food and beverage price index series. The findings suggest that the series level is non-stationary $(\mathrm{ADF}=-0.871, \mathrm{p}=0.955)$. However, the series was stationary in the first difference $(\mathrm{ADF}=-7.894, \mathrm{p}=0.01)$ at lag $12(\mathrm{ADF}=-6.307, \mathrm{p}=-0.01)$, and at differencing (1) of the seasonally differenced series ( $\mathrm{ADF}=-6.307, \mathrm{p}=-0.01)$. As a result, seasonal ARIMA (P, D, Q) model takes the value $\mathrm{D}=0$ while the non-seasonal ARIMA (p, d, q) model takes the value $\mathrm{d}=1$.

TABLE IV: UNIT ROOT TEST

\begin{tabular}{ccccc}
\hline \hline & Level & First Difference & $\begin{array}{c}\text { Seasonal Difference } \\
\text { at lag 12(SDL12) }\end{array}$ & $\begin{array}{c}\text { First Difference } \\
\text { of the SDL12 }\end{array}$ \\
\hline \hline ADF & -0.871 & -7.894 & -6.422 & -6.307 \\
P-value & 0.955 & $0.01^{* *}$ & $0.01^{* *}$ & $0.01^{* *}$ \\
\hline \hline
\end{tabular}

\section{E. Model Building}

Involves the process of examining the most appropriate SARIMA model as presented in this section.

\section{1) Model Indentification}

After identifying the series' stationarity, the order of both the seasonal and non-seasonal components of $\mathrm{AR}$ and MA processes that are suitable for the model is evaluated by inspecting the behavior of PACF and ACF. Fig. 3 depicts correlogram plots with seasonal lags ranging from 0 to 50 . The non-seasonal terms are determined by examining the initial lags. Non-seasonal MA terms are indicated by spikes in the ACF at short lags. Spikes in the PACF, on the other hand, indicate the possibility of non-seasonal AR terms at short lags. At lag 2, the early lags in the PACF tails are observed. As a result, we can calculate the non-seasonal MA (2) process. The PACF exhibits a notable spike at lag one, which then levels off until lags 12, 24 The spikes, however, fade with time. The pattern is followed by a tapering sequence in the ACF plot's lags. As a result, a non-seasonal AR (1) can fit a portion of the model. The patterns across lags that are multiples of $\mathrm{S}$ are inspected to determine the order of the seasonal component. The behaviors of the spikes about lags $12,24,36$, and so on are examined for monthly data. The ACF plot depicts a cluster of (negative) spikes near lags 12, 24, and 48. Positive spike clusters, on the other hand, are observed at lag 36. The PACF taper in $\mathrm{S}=12$ multiples; the PACF has notable lags at 12, 24 and 36 similar to the seasonal moving average (1) process. We can deduce an ARIMA $(1,1,0)(0,1,1) 12$ based on the ACF and PACF of the 12th differences (Fig. 4). 

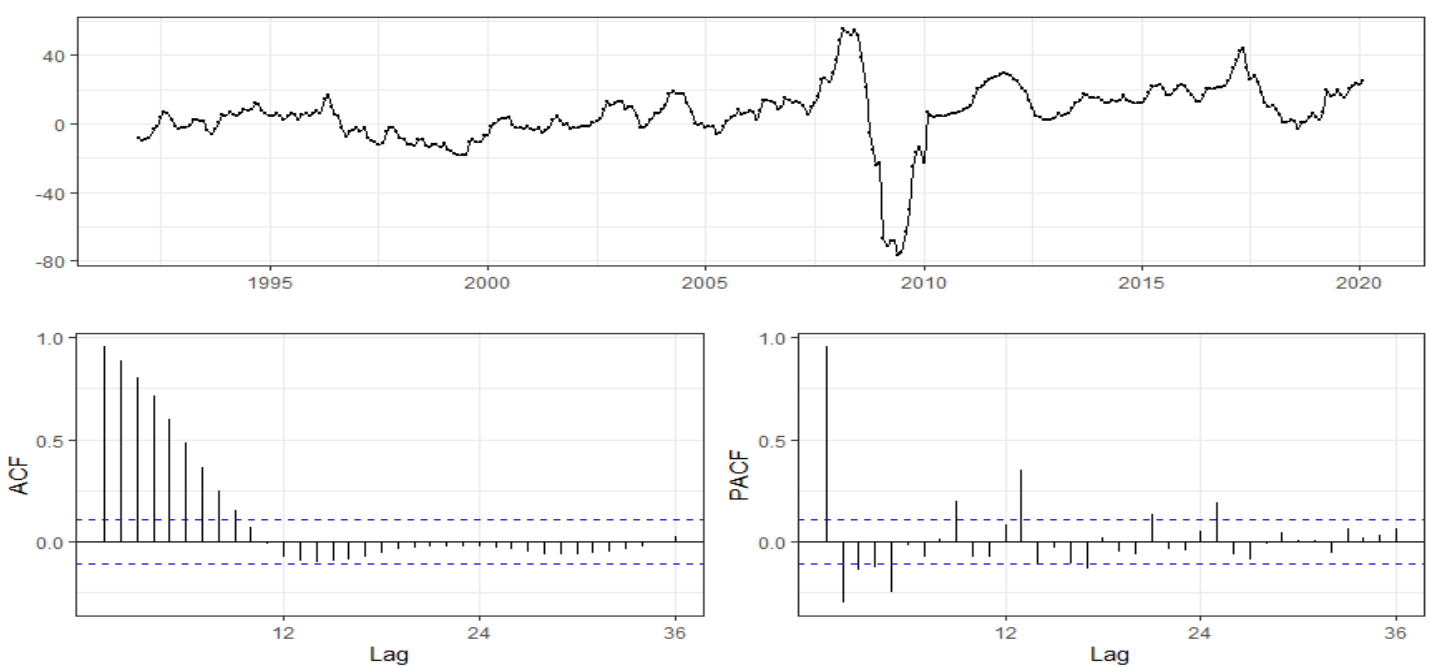

Fig. 3. The seasonally differenced series at lag 12 and its associated ACF and PACF.
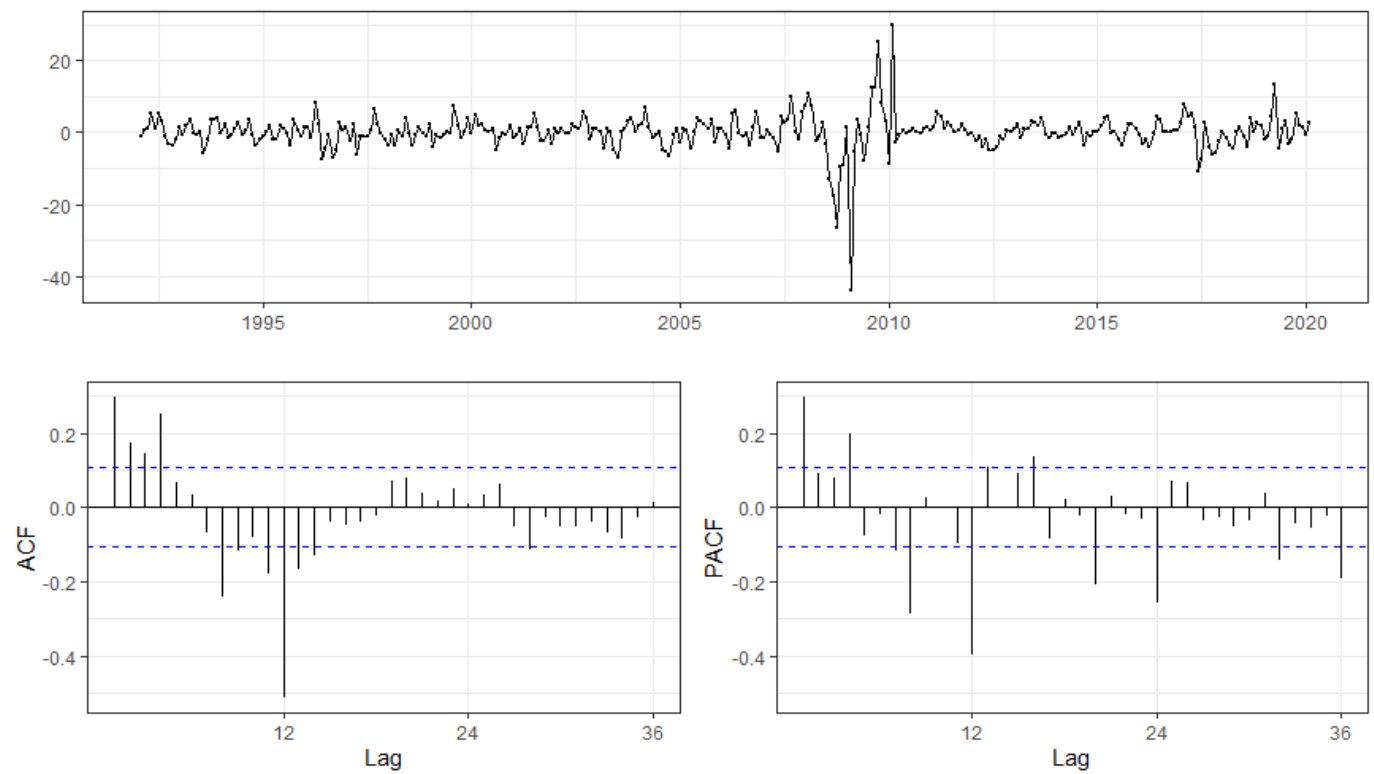

Fig. 4. AFC and PACF Plots of the First Difference of the Seasonally Differenced series at lag 12.

The parsimonious rule permits the researcher to choose the simplest model that sufficiently explains the series' behavior [29]. The study employed a grid search approach, where a combination of competing orders was examined to find a model that curtails one of the information criteria. Here, a range of parameter combinations is examined. To avoid fitting over the parametrized model, AIC and BIC help select the best model, in which the best model has the least value of these evaluation criteria [37]. Table V compares 17 estimated models based on AIC and BIC.

TABLE V: COMPARISON OF SARIMA MOdELS USING AIC AND BIC

\begin{tabular}{cccc}
\hline \hline SARIMA MODEL(Order) & S & AIC & BIC \\
\hline \hline$(0,1,2)(1,0,0)$ & 12 & 1860.968 & 1876.388 \\
$(0,1,2)(0,0,1)$ & 12 & 1860.969 & 1876.389 \\
$(0,1,2)(0,1,1)$ & 12 & 1820.349 & 1835.629 \\
$(0,1,3)(1,0,1)$ & 12 & 1858.767 & 1881.897 \\
$(1,1,0)(1,0,0)$ & 12 & 1858.565 & 1870.130 \\
$(1,0,0)(0,1,1)$ & 12 & 1818.174 & 1829.635 \\
$(1,1,0)(0,1,1)$ & 12 & 1818.174 & 1829.635 \\
$(1,1,1)(0,0,1)$ & 12 & 1859.084 & 1874.504 \\
$(1,1,1)(0,1,1)$ & 12 & 1818.153 & 1833.433 \\
$(1,1,2)(1,0,0)$ & 12 & 1860.906 & 1880.181 \\
$(2,1,0)(1,0,0)$ & 12 & 1859.302 & 1874.722 \\
$(2,1,1)(1,0,0)$ & 12 & 1859.700 & 1879.000 \\
$(2,1,1)(0,0,1)$ & 12 & 1859.721 & 1.185 \\
$(3,1,0)(1,0,0)$ & 12 & 1861.709 & 189.996 \\
$(3,1,1)(1,0,0)$ & 12 & 1862.871 & 1884.840 \\
$(3,1,1)(0,0,1)$ & 12 & 1862.965 & 1886.001 \\
$(0,1,3)(1,0,0)$ & & 12.240 \\
\hline \hline
\end{tabular}


The findings in Table four show that the SARIMA $(1,1,1)(0,1,1) 12$ model is most suitable candidate based on Akaike (AIC) and Bayesian (BIC) criteria. The non-seasonal specifications of AR (1), differencing (1), and MA (1) are given first, then follows the seasonal specifications of SAR (0), seasonal differencing (1), SMA (1) and the span of seasonality $(\mathrm{h}=12)$.

\section{2) Model Estimation}

The estimation of the model's coefficients employs the maximum likelihood estimation method, following the [31] time series modeling. The output in Table VI displays the estimated coefficients of the SARIMA $(1,1,1)(0,1,1) 12$ model.

\begin{tabular}{ccccc}
\multicolumn{7}{c}{ TABLE VI: TABLE OF COEFFICIENTS } \\
\hline \hline Estimate & SE & t-value & p-value \\
\hline \hline AR1 & 0.5755 & 0.1642 & 3.5053 & 0.0005 \\
MA1 & -0.3045 & 0.1955 & -1.5577 & 0.1202 \\
SMA1 & -0.921 & 0.0431 & -21.3749 & 0.0000 \\
\hline \hline
\end{tabular}

$\sigma^{2}=11.78: \log$ likelihood $=-905.08$, AIC $=1818.15, \mathrm{BIC}=1833.4$

The non-seasonal AR1 and SMA1 components were statistically significant at a $1 \%$ level (all p-values $<0.01)$. The coefficient of the AR component is average (0.576), given that the coefficient ranges from 0 to 1 . The finding implies that the FBPI series returns to its mean relatively moderately. The non-significance of the non-seasonal MA component points out that past periodic shocks to FBPI in Kenya do not significantly explain the present price index levels. The results are congruent to those of Nyoni [46], who found that the non-seasonal AR explains the current CPI in Kenya, whereas the MA did not. However, their study used the ARIMA model, which does not capture seasonality. By extension, the current study demonstrates seasonality component incorporates the MA component only. Since it is close to 1, the seasonal shocks are infrequent. As shown by the visual shocks in the FBPI series, the two peaks occurred during the 1998/9 and 2008/09 financial crises.

From the Table VI, the SARIMA $(1,1,1)(0,1,1)_{12}$ model can be expressed as:

$$
X_{t}=0.5755-0.3045 e_{t-1}-0.921 \epsilon_{t-1}
$$

where; $X_{t}$ is the CPI series, $X_{t-1}$ is the non-seasonal AR process of order $1, e_{t-1}$ is the non-seasonal MA process of orders 1 , and $\epsilon_{t-1}$ is the SMA process of order 1 .

\section{3) Diagnostics Checks of the Model}

The estimated best fit model must be examined to ascertain if it sufficiently represents the FBPI data in Kenya. As a result, diagnostic checks were run on the residuals to see if they meet the normality assumption and autocorrelation. The models' standardized residuals ought to resemble an independently and identically distributed sequence with mean zero and constant variance. The residual plot demonstrates that the standardized residual plots have a constant variance. The residuals are almost normally distributed, according to the normal Q-Q plots, because they lie along the 45 line (Fig. 5).
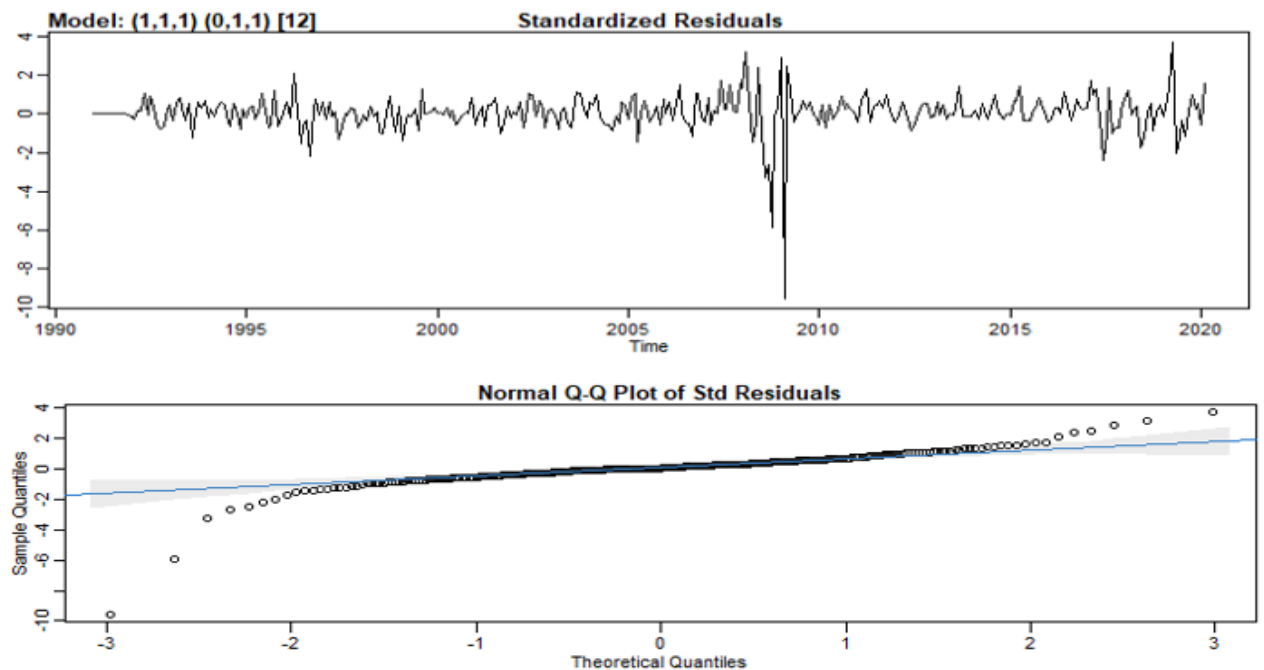

Fig. 5. Standardized Residuals Plots of ARIMA $(1,1,1)(0,1,1) 12$ model.

The ACF plot shows There is no serial correlation amongst each lagged values because the residuals have no notable autocorrelation (Fig. 6). The residual ACF plots do not show enough existence of 
significant spikes, implying that the residuals are white noise. Because the associated Q-statistics have large p-values (greater than 0.05), the Ljung-Box Q-test revealed that the residuals are purely white noise. As a result, no serial autocorrelation and partial autocorrelation coefficients between residuals at different lag times are statistically insignificant up to lag 7. The findings imply that residuals errors are not correlated and thus do not require ARCH class of models.
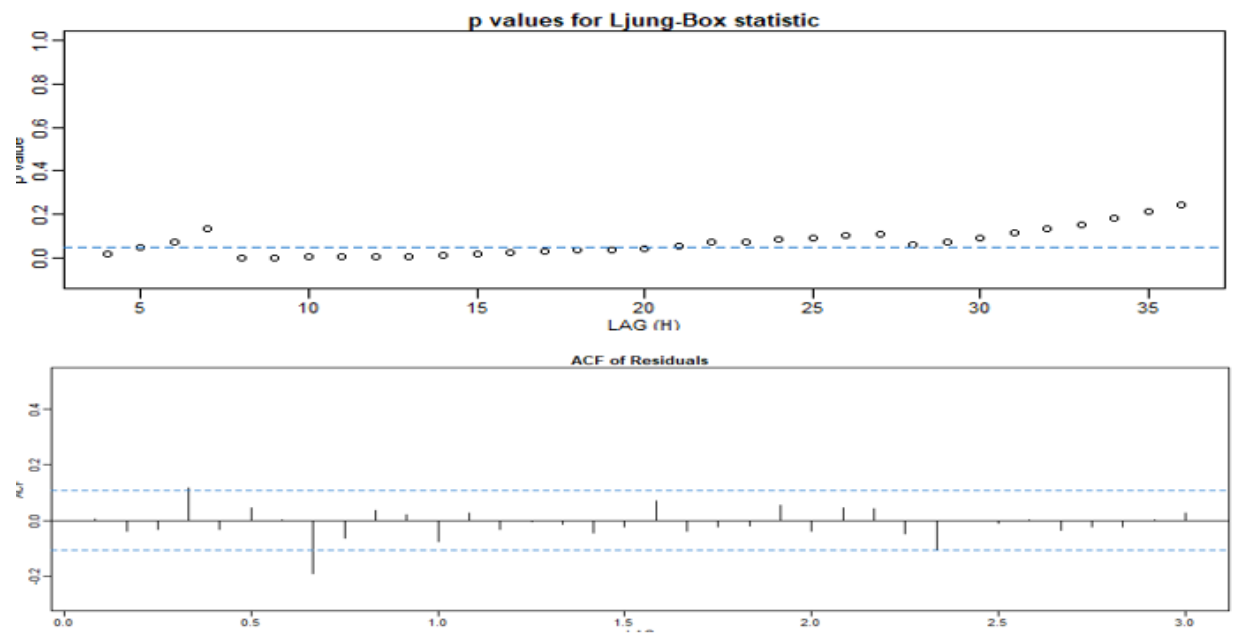

Fig. 6. The Ljung-Box Q-test and ACF of residuals.

\section{4) Model Accuracy Test}

The accuracy-test summarized in Table VII was applied in determination of the model's accuracy in making an h-step ahead prediction. The model can be considered as a better fit since it is much more accurate in terms of MAPE of the forecast of $1.62 \%$ compared to those of [47], who fitted SARIMA $(1,1$, 1) $(0,1,2) 12$ to monthly rainfall data for Embu County with MAPE of $9.0 \%$ in its 2-year period predictive ability.

TABLE VII: MODEL ACCURACY TEST

\begin{tabular}{cc}
\hline \hline Parameter & Value \\
\hline \hline RMSE & 3.3678 \\
MAE & 2.0013 \\
MAPE & 1.6238 \\
\hline
\end{tabular}

Note: The accuracy metrics values result from the in-sample prediction errors from the fitted model.

Compared to past studies that employed SARIMA to fit the general inflation show a slight but comparable disparity. For instance, the fitted model performed better than those of [17], who fitted SARIMA $(0,1,0)(0,0,1) 12$ to inflation rates in Kenya applying quarterly data for the year 1981 to 2013 in terms of MAPE (3.945) only. In Uganda, Kinene [48] SARIMA (1,1,0) $(1,0,1) 12$ minimized the prediction errors better than the current study's fitted model (RMSE $=1.2191)$. In another Kenyan study, the SARIMA $(0,1,0)(0,0,1) 4$ model of [23], which best-fitted inflation rate quarterly data from 1981 to 2013 , has predictive ability metrics as follows: RMSE (0.2871), MAPE (3.9456), and MAE (0.2369). The disparity can also be explained by the different time frames and frequency of data. For instance, [17] used quarterly data, and [48] used a one-step-ahead forecast, unlike the current study, which used two-step ahead period forecasts. Nonetheless, the fitted and the initial data sets, as shown in Fig. 7, are in synchrony, indicating that the model is suitable for the data.

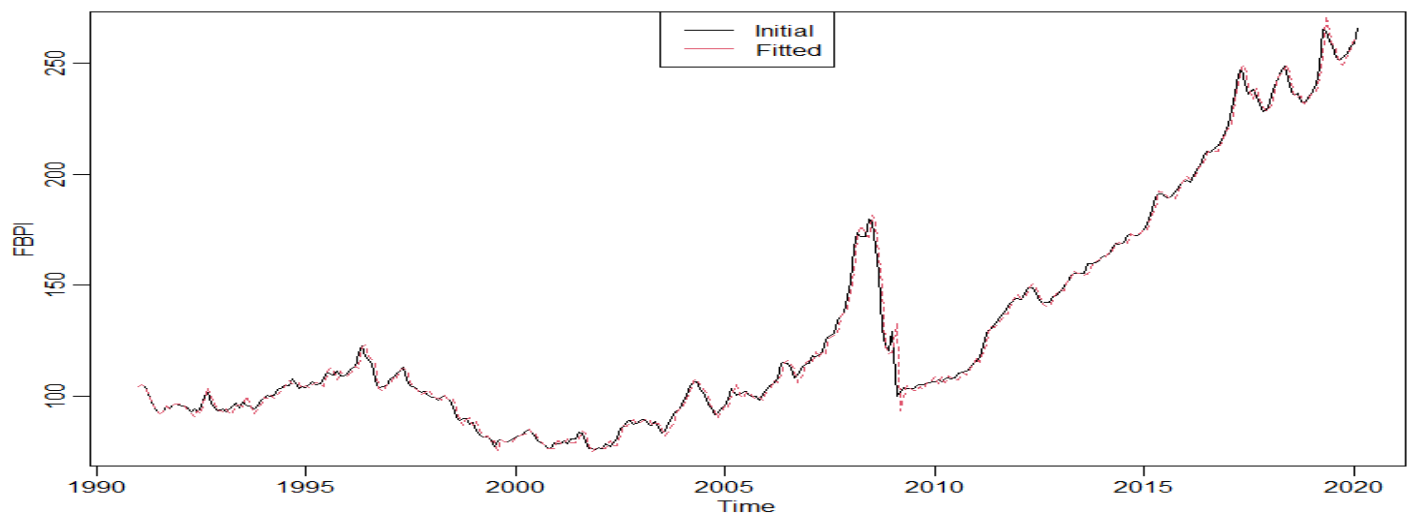

Fig. 7. Initial and Fitted FBPI data. 


\section{F. Forecasting}

The practice of forecasting inflation has typically been regarded as a vital input in monetary policymaking [43]. It aids in planning and decision-making by providing awareness into the future based on the past and existing behavior of given observed values. As a result, the final model was applied to fit h-step ahead predictions. The Forecasts for the next 24 months, March 2020 to March 2022, with the 95 percent confidence interval (one) and two standard error prediction bounds overlaid in grey (Fig. 8).

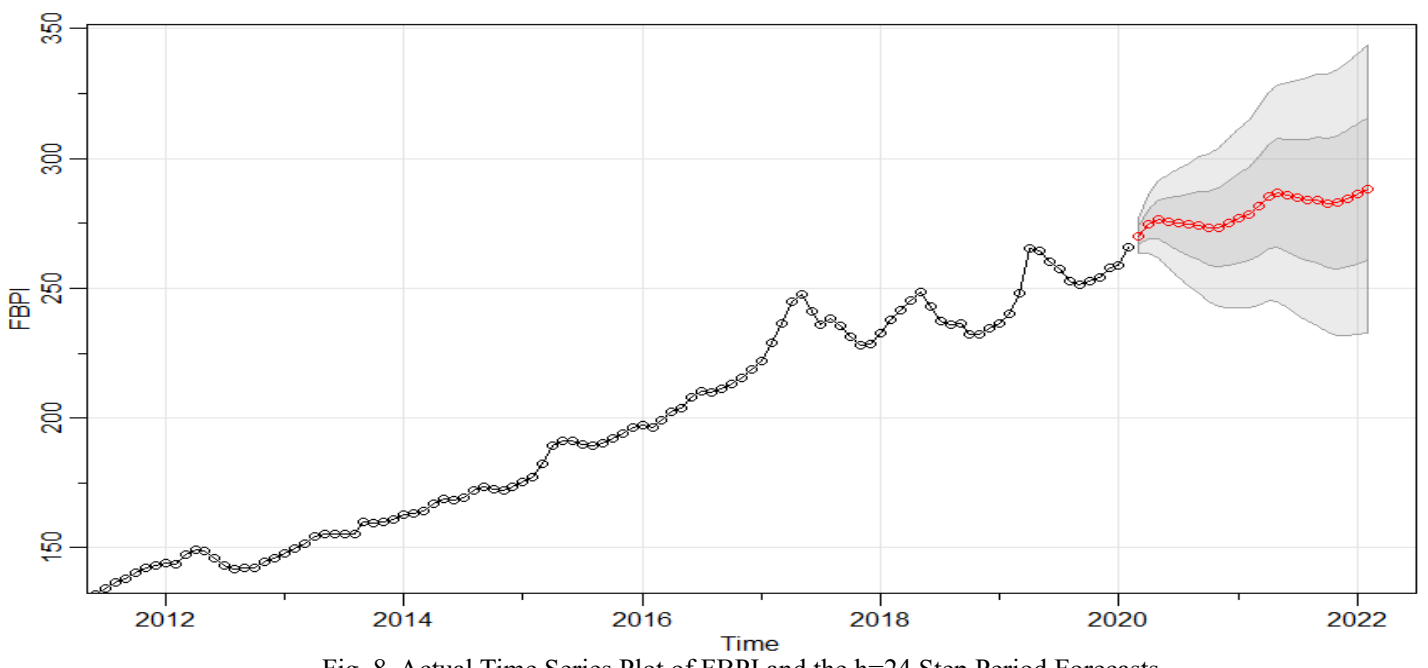

Fig. 8. Actual Time Series Plot of FBPI and the $\mathrm{h}=24$ Step Period Forecasts.

Note: The forecasted values are red with the 95 percent and 99 percent Prediction bounds.

The forecasts show a decreasing trend from 276.46 in May 2020 to about 273.1289 in October 2020 (273.13) (Table IX). After that, the model prediction shows another upward trend from 273.93 in November 2020 to 286.66 in May 2021. The subsequent downfall from June 2021 (285.740) ended in October 2021 (282.75), followed by an upward trend to the end of the forecast period in February 2021 (288.18). Overall, the food price index appears to have increased trend over time. The findings support past studies, such as the study done by [17], [23], [46], [49], whose forecasts indicated that inflation is more likely to rise in the future in Kenya.

TABLE VIII: FORECASTS (24-STEP AHEAD)

\begin{tabular}{|c|c|c|c|c|c|c|}
\hline Month & 2020 (Estimates) & $\mathrm{SE}$ & 2021 (Estimates) & SE & 2022 (Estimates) & $\mathrm{SE}$ \\
\hline Jan & & & 276.88 & 17.25 & 286.47 & 27.02 \\
\hline Feb & & & 278.58 & 18.14 & 288.17 & 27.7 \\
\hline March & 270.17 & 3.43 & 281.6 & 19.07 & & \\
\hline Apr & 274.64 & 5.55 & 285.28 & 19.99 & & \\
\hline May & 276.46 & 7.41 & 286.66 & 20.87 & & \\
\hline June & 275.8 & 9.06 & 285.74 & 21.73 & & \\
\hline July & 274.98 & 10.54 & 284.77 & 22.56 & & \\
\hline Aug & 274.42 & 11.88 & 284.13 & 23.36 & & \\
\hline Sept & 274.3 & 13.11 & 283.95 & 24.14 & & \\
\hline Oct & 273.13 & 14.25 & 282.75 & 24.89 & & \\
\hline Nov & 273.41 & 15.31 & 283.02 & 25.62 & & \\
\hline Dec & 274.93 & 16.31 & 284.53 & 26.33 & & \\
\hline
\end{tabular}

\section{CONCLUSION}

Forecasting is common practice and a vital aspect of monetary policymaking. An evidence-based and valid policy option should bank on reliable forecasts that can be realized if the most suitable model with higher predictive accuracy is used. Following the Box-Jenkins model building approach, this study has demonstrated that SARIMA $(1,1,1)(0,1,1) 12(\mathrm{AIC}=1818.15, \mathrm{BIC}=1833.4, \mathrm{MAE}=2.00 \% \mathrm{MAPE}=$ $1.62 \%$, MASE $=0.869 \%$ ). All the prediction metrics lies below the acceptable range of 5 percent thus the model was best fit for food and beverage and price index in Kenya. Besides, the 24-step ahead forecasts depicted that food and beverage prices seem to be volatile. However, SARIMA models are unstable for long time period forecasts and can only be used in making short-term forecasts. The model is relevant to the Monetary Policy Committee who carries out the periodic evaluation of price indices to direct their policy actions, such as price controls. 


\section{ACKNOWLEDGMENT}

I would like to give thanks to the Almighty God for the gift of life, strength and wisdom throughout this master's program. Special thanks to my supervisors Dr. Dennis Muriithi and Dr. Adolphus Wagala for their overwhelming guidance and commitment throughout my study.

\section{FUNDING}

The study received no external/internal funding.

\section{CONFLICT OF INTEREST}

The authors declare no conflict of interests.

\section{REFERENCES}

[1] Smith JL. World oil: market or mayhem? Journal of Economic Perspectives. 2009; 23(3): 145-64.

[2] Misati RN, Nyamongo EM, Mwangi I. Commodity price shocks and inflation in a net oil-importing economy. OPEC Energy Review. 2013; 37(2): 125-48.

[3] Maxwell Fry DJ, Lavan Mahadeva SR, Sterne G. Key issues in the choice of monetary policy framework. Monetary policy frameworks in a global context. 2000; 2 .

[4] Ngwen N, Amba Oyon CM, Mbratana T. Government expense, Consumer Price Index and Economic Growth in Cameroon.

[5] Knaut A, Paschmann M. Price volatility in commodity markets with restricted participation. Energy Economics. 2019; 81: 3751 .

[6] Nathan MM, Jagongo A. Monetary policy tools and inflation in Kenya. Money. 2017; 7(1): 86-97.

[7] Laxton D, Rose D, Scott AM. Developing a Structured Forecasting and Policy Analysis System to Support Inflation-Forecast Targeting (IFT).

[8] CBK. Central Bank of Kenya. Monetary Policy Statement, CBK, Nairobi, 2017.

[9] Headey D, Fan S. Reflections on the global food crisis: How did it happen? How has it hurt? And how can we prevent the next one?. International Food Policy Research Institute. 2010.

[10] Brida JG, Garrido N. Tourism forecasting using SARIMA models in Chilean regions. International Journal of Leisure and Tourism Marketing. 2011; 2(2): 176-90.

[11] Stoklasová R. Model of the unemployment rate in the Czech Republic. InProceedings of 30th international conference on mathematical methods in economics 2012; 836-841.

[12] Nasiru S, Sarpong S. Empirical Approach to Modelling and Forecasting Inflation in Ghana. Current Research Journal of Economic Theory. 2012; 4(3): 83-87.

[13] Adanacioglu H, Yercan M. An analysis of tomato prices at wholesale level in Turkey: an application of SARIMA model. Custos e@gronegócio on line. 2012; 8(4): 52-75.

[14] Kibunja HW, Kihoro JM, Orwa GO, Yodah WO. Forecasting precipitation using SARIMA Model: A case study of Mt. Kenya Region.

[15] Kumar M, Anand M. An application of time series ARIMA forecasting model for predicting sugarcane production in India. Studies in Business and Economics. 2014; 9(1): 81-94.

[16] Etuk EH. An additive Sarima model for daily exchange rates of the Malaysian Ringgit (MYR) and Nigerian Naira (NGN). International Journal of Empirical Finance. 2014; 2(4): 193-201.

[17] Gikungu SW, Waititu AG, Kihoro JM. Forecasting inflation rate in Kenya using SARIMA model. American Journal of Theoretical and Applied Statistics. 2015; 4(1): 15-18.

[18] Dritsaki C. Forecast of SARIMA models: An application to unemployment rates of Greece. American Journal of Applied Mathematics and Statistics. 2016; 4(5): 136-48.

[19] Mutwiri RM. Forecasting of tomatoes wholesale prices of Nairobi in Kenya: time series analysis using SARIMA model. Journal of Statistical Distributions and Applications. 2019; 5(3): 46-53.

[20] Suleman N, Sarpong S. Empirical approach to modeling and forecasting inflation in Ghana. Current Research Journal of Economic Theory. 2012; 4(3): 83-87.

[21] Boateng FO, Amoah-Mensah J, Anokye M, Osei L, Dzebre P. Modeling of tomato prices in Ashanti region, Ghana, using seasonal autoregressive integrated moving average model. Journal of Advances in Mathematics and Computer Science. 2017: $1-3$.

[22] Otu OA, Osuji GA, Opara J, Mbachu HI, Iheagwara AI. Application of Sarima models in modelling and forecasting Nigeria's inflation rates. American Journal of Applied Mathematics and Statistics. 2014; 2(1): 16-28.

[23] Gikungu SW, Waititu AG, Kihoro JM. Forecasting inflation rate in Kenya using SARIMA model. American Journal of Theoretical and Applied Statistics. 2015; 4(1): 15-18.

[24] Omane-Adjepong M, Oduro FT, Oduro SD. Determining the better approach for short-term forecasting of ghana's inflation: Seasonal ARIMA Vs holt-winters. International Journal of Business, Humanities and Technology. 2013; 3(1): 69-79.

[25] Lidiema C. Modelling and Forecasting Inflation Rate in Kenya Using SARIMA and Holt-Winters Triple Exponential Smoothing. American Journal of Theoretical and Applied Statistics. 2017; 6(3): 161-169.

[26] Baeta FD, Tumaku J, Ahiave EK. The optimal forecast model for Ghanas inflation: A stochastic approach. Journal of Economics and International Finance. 2019; 11(2): 15-23.

[27] Verbesselt J, Hyndman R, Newnham G, Culvenor D. Detecting trend and seasonal changes in satellite image time series. Remote Sensing of Environment. 2010; 114(1): 106-115.

[28] Wang W, Niu Z. Time series analysis of NASDAQ composite based on seasonal ARIMA model. In2009 International Conference on Management and Service Science 2009 Sep 20 (pp. 1-4). IEEE.

[29] Chen KY, Wang CH. A hybrid SARIMA and support vector machines in forecasting the production values of the machinery industry in Taiwan. Expert Systems with Applications. 2007; 32(1): 254-264.

[30] Shumway RH, Stoffer DS. Time series regression and exploratory data analysis. Time Series Analysis and Its Applications: With R Examples. 2006: 48-83.

[31] Box GE, Jenkins GM, Reinsel GC, Ljung GM. Time series analysis: forecasting and control. 2015. 
[32] RStudio Team. RStudio: Integrated Development Environment for R [Internet]. Boston, MA; 2015. Available from: http://www.rstudio.com/

[33] Dickey DA, Fuller WA. Distribution of the estimators for autoregressive time series with a unit root. Journal of the American Statistical Association. 1979; 74(366a): 427-31.

[34] Box GEP, Jenkins GM, Reinsel GC. Time series analysis, forecasting and control. Englewood Clifs. 1994.

[35] Vandekerckhove J, Matzke D, Wagenmakers EJ. Model comparison and the principle of parsimony. eScholarship, University of California; 2014.

[36] Nkoro E, Uko AK. Autoregressive Distributed Lag (ARDL) cointegration technique: application and interpretation. Journal of Statistical and Econometric Methods. 2016; 5(4): 63-91.

[37] Phung D, Huang C, Rutherford S, Chu C, Wang X, Nguyen M, et al. Identification of the prediction model for dengue incidence in Can Tho city, a Mekong Delta area in Vietnam. Acta Tropica. 2015; 141: 88-96.

[38] Akaike H. On the Entropy Maximization Principle."PR Krishniah (ed.), Applications of Statistics: 27-41. 1977.

[39] Hyndman RJ, Khandakar Y. Automatic time series for forecasting: the forecast package for R (No. 6/07). Clayton VIC, Australia: Monash University, Department of Econometrics and Business Statistics. 2007.

[40] Schwarz G. Estimating the dimension of a model. The Annals of Statistics. 1978: 461-4.

[41] McLeod AI. On the distribution of residual autocorrelations in Box-Jenkins models. Journal of the Royal Statistical Society: Series B (Methodological). 1978; 40(3): 296-302.

[42] Horowitz JL, Lobato IN, Nankervis JC, Savin NE. Bootstrapping the Box-Pierce Q test: a robust test of uncorrelatedness. Journal of Econometrics. 2006; 133(2): 841-62.

[43] Fisher JD, Liu CT, Zhou R. When can we forecast inflation?. Economic Perspectives-Federal Reserve Bank of Chicago. 2002; 26(1): 32-44

[44] Durevall MD, Ndung'u NS. A dynamic model of inflation for Kenya, 1974-1996. International Monetary Fund, 1999.

[45] Andrle M, Berg MA, Morales MR, Portillo R, Vlcek MJ. Forecasting and monetary policy analysis in low-income countries: Food and non-food inflation in Kenya. International Monetary Fund. 2013.

[46] Nyoni T. Modeling and forecasting inflation in Kenya: Recent insights from ARIMA and GARCH analysis. Dimorian Review. 2018; 5(6): 16-40.

[47] Filder TN, Muraya MM, Mutwiri RM. Application of seasonal autoregressive moving average models to analysis and forecasting of time series monthly rainfall patterns in Embu County, Kenya. Asian Journal of Probability and Statistics. 2019: 1-15.

[48] Kinene A. FORECASTING OF THE INFLATION RATES IN UGANDA: A COMPARISON OF ARIMA, SARIMA AND VECM MODELS. 2016.

[49] Uwilingiyimana C, Munga'tu J, Harerimana JD. Forecasting Inflation in Kenya Using Arima-Garch Models. International Journal of Management and Commerce Innovations. 2015; 3(2): 15-27. 